Approximation Theory and Functionat Analysis

J.B. Prolla (ed.)

(c) North-Holland Publishing Company, 1979

\title{
A REMARK ON VECTOR-VALUED APPROXIMATION ON COMPACT SETS, APPROXIMATION ON PRODUCT SETS, AND THE APPROXIMATION PROPERTY
}

\author{
KLAUS - D. BIERSTEDT \\ FB 17 der GH, Mathematik, D2-228 \\ Warburger Str. 100, Postfach 1621 \\ D-4790 Paderborn \\ Germany (Fed. Rep.)
}

\section{INTRODUCTION}

After Grothendieck [21], a locally convex (l.c.) space $E$ is said to have the approximation property (for short, a.p.) if and only if the identity $i d_{E}$ of $E$ can be approximated uniformly on every precompact subset of $\mathrm{E}$ by continuous linear operators from $\mathrm{E}$ into E of finite rank (i.e. with finite dimensional range). Many "concrete" l.c. spaces are known to have the a.p., but a counterexample of Enflo (1972), with subsequent refinements due to Figiel, Davie, and Szankowski, shows that there are even closed subspace of $\ell^{p}$ without a. p. for each $p \geq 1, p \neq 2$.

In connection with the a.p., a criterion due to L. Schwartz [26] is very useful: Schwartz introduces for two $\ell . c$.spaces $E$ and $F$ their $\varepsilon$-product by

$$
E \in F:=\mathcal{L}_{e}\left(F_{C}^{\prime}, E\right)
$$

where $F_{C}^{\prime}$ is the dual of $F$ with the topology of uniform convergence on precompact subsets of $F$ and where the subscript $e$ on the space 
$\mathcal{L}\left(F_{C^{\prime}}^{\prime} E\right)$ of all continuous linear operators from $F_{C}^{\prime}$ into $E$ indicates the topology of uniform convergence on the equicontinuous subsets of $F^{\prime}$.

If $E$ and $F$ are quasi-complete, one can easily show $E \varepsilon F \cong F \varepsilon E$, and the $\varepsilon$-product $E \in F$ of two complete spaces $E$ and $F$ is complete (cf. [26]). Moreover, the e-tensor product $E \otimes_{\varepsilon} F$ of Grothendieck [21] is a topological subspace of $E \varepsilon F$. We can now formulate Schwartz's criterion for the a.p. ([26], Proposition 11, cf. also [3], I, 3.9, and (8]) :

THEOREM (L. Schwartz): The quasi-complete l.c. space $\mathrm{E}$ has the a.p. if and only if $E \otimes F$ is dense in $E \in F$ for each lquasi-1 complete l.c. space $F$ lor, equivalently, for each Banach space Fl. So if $\mathrm{E}$ and $F$ are complete l.c. spaces such that $E$ or $F$ has the a.p., we get: $E \in F=E \check{\otimes}_{\varepsilon} F$, the completion of the $\varepsilon$-tensor product $E \otimes_{E} F$ (which we will also call, for short, complete $\varepsilon$-tensor product).

In fact, the applications of this theorem, say, in the case of function spaces $\mathrm{E}$ derive from the remark that the "abstract"operator space $E \in F$ can usually be identified with a "concrete" space of F-valued functions "of type $E$ ". And $E \otimes_{\varepsilon} F$ is the space of "corresponding" functions with finite dimensional ranges in $F$. Hence a proof of the a.p. of $E$ is then equivalent to the approximation of certain F-valued functions by functions with values in finite dimensional subspaces of $F$ for every (quasi-) complete $\ell$.c. space $F$ or only for every Banach space $F$, a result which is of interest in both directions.

In this article, we will give some (rather simple) new examples of how to apply Schwartz's theorem to function spaces more general and $A(K)$ on compact subsets $K$ of $\mathbb{C}^{N}(N \geq 1)$. More precisely, we deal here with spaces of continuous functions on a compact set $K$ which 
either are uniformly approximable by functions belonging, on open sets $U$ containing $K$, to a given subsheab $F$ of the sheaf $c$ of all continuous functions or have restrictions belonging to $F$ on the interior $\stackrel{\circ}{K}$ of $K$.

The general situation is the subject of sections 1 and 2 . In section 1 , the vector-valued case is considered, while section 2 deals with "slice product" - results (on product sets). Finally, in section 3 , we look at some of the motivating examples and survey the known results (and their relations) in this case.

So, in a sense, this paper is based on a generalization of the author's old article [2] and motivated, among other things, by the more recent article [27] of $\mathrm{N}$. Sibony: We show the connection of some of Sibony's results with topological tensor product theory and with the a.p. of the spaces of scalar functions in question. The results of this paper will be combined with the technique of "localization" of the a.p. for subspaces of weighted Nachbin spaces (cf.[5] and [10]) in a subsequent paper to yield new examples of function spaces "of mixed type" with a.p. and to demonstrate applications of the local ization procedure in some concrete cases.

ACKNOWLEDGEMENT: The author gratefully acknowledges support under the GMD/CNPq agreement during his stay at UNICAMP July-September 1977 without which it would not have been possible to attend this conference in Campinas. I would also like to thank J. B. Prolla for his constant interest in my contribution to these Proceedings. As everybody can see immediately, part of the results in this article dates (at least) back to the time when the joint publication [10] was prepared. So the author thanks B. Gramsch and R. Meise for many helpful conversations and remarks in this connection. 
1. THE GENERAL VECTOR-VALUED CASE

Let $\mathrm{X}$ be a completely regular (Hausdorff) topological space and $F$ a closed locally convex (l.c.) subsheaf of the sheaf $C_{X}$ of all continuous (real or complex valued) functions on $x, i . e$. , for each open subset $u$ of $x, F(u)$ is a closed topological linear subspace of $C(u)$ with the compact-open topology co. In fact, it would be sufficient to require $F$ to be a presheaf only, and we prefer to use presheaf notation throughout this paper. (For some of our notation compare [9] and [10]. A sheaf $F$ as above was called "sheaf of F-morphic functions" in [9].)

Let $E$ always denote a quasi-complete locally convex (Hausdorff) space (over $\mathbb{R}$ or $\mathbb{C}$ ). We will always assume that $X$ is a $k_{\mathbb{R}}$-space, i.e. that any function $f: X \rightarrow \mathbb{R}$ (or, equivalently, any function $\mathrm{f}: \mathrm{X} \rightarrow \mathrm{Y}, \mathrm{Y}$ any completely regular space) is continuous if and only if the restriction of $f$ to each compact subset of $X$ is continuous. (Each locally compact or metrizable space, and, more generally, each $k$-space is also a $k{ }_{\mathbb{R}}$-space.) Then each open $u \subset x$ is again a ${ }^{K}{ }_{R}$-space, cf. Blasco $[12]$, and hence the sheaves $C_{X}$ and $F$ are complete, i.e. the spaces $(c(u), c o)$ and $F(u)$ are complete for each open
$u \subset x$.

Under these assumptions, there exists (cf. [10], 1.5) the "E-valued sheaf $F^{E}$ of $F^{\prime \prime}$, namely, for any open $u$ in $x$,

$$
\begin{aligned}
F^{E}(U)= & E \in F(U) \\
= & \text { the space of all continuous E-valued functions } f \text { on } U \\
& \text { which satisfy } e^{\prime} \circ f \in F(U) \text { for each } e^{\prime} \in E^{\prime} \text {, endowed } \\
& \text { with the topology co of uniform convergence on corpact } \\
& \text { subsets of } u(\operatorname{cf} .[3] \text { and }[5]),
\end{aligned}
$$

and the cononical restriction mappings of the sheaf $F^{E}$ are just the 
ordinary restrictions of functions. $F^{E}$ is a clased subsheaf of the sheaf $\mathrm{C}_{\mathrm{X}}^{\mathrm{E}}$ of all continuous E-valued functions on $\mathrm{X}$.

In our definition and in some of our results below, it may be helpful to keep the following motivating examples of sheaves $F$ of F-morphic functions in mind (cf. also [9] and [10] for more examples):

1. EXAMPLES: (i) $\mathrm{X}=$ complex monifold or just $\mathbb{C}^{\mathrm{N}}$ (iN $\geq 1$ ), $F=0=$ sheaf of holomorphic functions on $x$,

(ii) $\mathrm{X}$ open in $\mathbb{R}^{\mathrm{n}}(\mathrm{n} \geq 1), \mathrm{L}=\mathrm{P}(\mathrm{x}, \mathrm{D})$ a (linear) hypoelliptic differential operator with $C^{\infty}$-coefficients, and $F=N_{L}=$ sheaf of null solutions of $L$, i.e. $N_{L}(U)=\left\{f \in C^{\infty}(U) ;(L \mid U) f \equiv 0\right\}$ for any any open $u$ in $x$. (The closed graph theorem for Fréchet spaces implies that, on $N_{L}(u)$, the topologies induced by $\mathrm{C}^{\infty}(u)$ and by co coincide and hence that $N_{L}(u)$ is a closed topological linear subspace of $(\mathrm{C}(U), \mathrm{co})$.

Especially, the sheaf $\mathcal{H}$ of harmonic functions on $\mathbb{R}^{\mathrm{n}}$ satisfies all assumptions of 1. (ii) above, and also the "harmonic sheaves" of abstract potential theory are sheaves of F-morphic functions. A11 the sheaves of example I. are (FN)-sheaves.

2. DEFINITION: For a compact subset $K$ of $X$, we define:

(i) $\mathrm{C}(\mathrm{K}, \mathrm{E}):=$ the space of all continuous $\mathrm{E}$-valued functions on $K$ with the topology of uniform convergence on $K$,

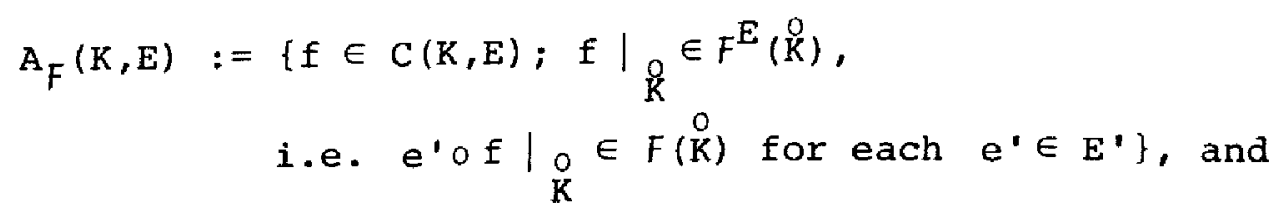

(iii) $H_{F}(K, E):=$ the closure in $C(K, E)$ of $\{f \in C(K, E)$; there exists an open neighbourhood $U$ of $K$ (depending on $f$ ) and a function $g \in F^{E}(u)$ li.e. $g: U \rightarrow E$ continuous and $e^{\prime} \circ g \in F(u)$ for any $\left.e^{\prime} \in E^{\prime}\right]$ such that $\left.\left.g\right|_{K}=f\right\}$. 
$\mathrm{H}_{F}(\mathrm{~K}, \mathrm{E}\rangle \subset \mathrm{A}_{F}(\mathrm{~K}, \mathrm{E})$ holds, and both are closed subspaces of $\mathrm{C}(\mathrm{K}, E)$ which we endow with the topology of uniform convergence on $K$ (induced by $C(K, E))$. If $E=\mathbb{R}$ or $\mathbb{C}$, we write $C(K), A_{F}(K)$, and $H_{F}(K)$, respec tively.

Now, of course, if $E$ is complete, all the spaces $C(K, E), A_{F}(K, E)$, and $H_{F}(K, E)$ are complete, too. The equation $C(K, E)=E \varepsilon C(K)$ for quasi-complete $\mathrm{E}$ is well-known (cf. [3]), and, once this equation is well-understood, the proof of the first part of the following result is clear (see e.g. [3] or [5] for a description of $E \in F, F$ an arbitrary subspace of $\mathrm{C}(\mathrm{K})$, from which our result below is easily derived, too) :

3. THEOREM: (1) $A_{F}(K, E)=E \varepsilon A_{F}(K)$.

(2) Hence $A_{F}(K, E)=E \check{\otimes}_{E} A_{F}(K)$ holds for all complete $\ell . c$. Ior, equivalently, for all Banachl spaces $E$ if and only if $\mathrm{A}_{F}(\mathrm{~K})$ has the a.p.

For the second part of 3, Schwartz's criterion for the a.p. (in the introduction) is needed. In other words, ${ }^{A} F(K)$ has the a.p. if and only if, for arbitrary Banach space $E$, each function $f \in C(K, E)$ with $e^{\prime}$ of $\left.\right|_{k} \in F(\stackrel{0}{K})$ for any $e^{\prime} \in E^{\prime}$ may be approximated, uniformly on $k$, by continuous functions $g$ on $k$ with values in finite dimensional subspaces of $E$ that satisfy $\left.e^{\prime} \circ g\right|_{K} ^{O} \in F(K)$, too, and hence
have the form

$$
g(x)=\sum_{i=1}^{n} e_{i} g_{i}(x) \quad \text { for all } \quad x \in K ;
$$

$n \in \mathbb{N}$ finite (depending on $g$ ), $e_{i} \in E$, and $g_{i} \in A_{F}(K), i=I, \ldots, n$. possible by the a.p. of $\mathrm{C}(\mathrm{K})$ and by with $\mathrm{g}_{i}$ only $\in \mathrm{C}(\mathrm{K})$ is always for complete l.c. E.) 
As to the $\mathrm{a}, \mathrm{p}$. Of the corresponding space $\mathrm{H}_{F}(\mathrm{~K})$, the situation there is, in some sense, just the opposite:

4. THEOREM: We assume that, for some basis ut of neighbourhoods of $\mathrm{K}, F(u)$ has the a.p. for each $u \in U$. [For (1) below, we could also assume instead that $\mathrm{E}$ has the a.p.l

(1) Then $E \otimes_{\varepsilon}{ }_{F}(K)$ is a dense topological linear subspace of $\mathrm{H}_{F}(\mathrm{~K}, \mathrm{E})$, and hence.

$$
\mathrm{H}_{F}(\mathrm{~K}, \mathrm{E})=E \check{\otimes}_{\varepsilon} \mathrm{H}_{F}(\mathrm{~K})
$$

holds whenever $\mathrm{E}$ is complete.

(2) Consequently $\mathrm{H}_{F}(\mathrm{~K})$ has the a.p. if and only if, for each complete l.c. lor each Banachl space E,

$H_{F}(K, E)=\left\{f \in C(K, E)\right.$; for each $e^{\prime} \in E^{\prime}$ and each $\varepsilon>0$ there exists an open neighbourhood $U=U\left(e^{\prime}, \varepsilon\right)$ of $\mathrm{K}$ and a function $g=g\left(e^{\prime}, \varepsilon\right) \in F(u)$ such that

$$
\left.\sup _{x \in \mathbb{K}}\left|\left(e^{\prime} \circ f\right)(x)-g(x)\right|<\varepsilon\right\} .
$$

PROOF: As $E \otimes_{\varepsilon} C(K)$ is a topological linear subspace of $C(K, E)$ and as the $\varepsilon$-tensor product preserves topological linear subspaces, only density of $E \otimes H_{F}(K)$ in $H_{F}(K, E)$ must be verified for the first assertion. So let $\mathrm{p}$ be a continuous seminorm on $E, \varepsilon>0$ and $f \in H_{F}(K, E)$. By definition, there exists an open set $U \supset K$ and $a$ function $g \in F^{E}(u)$ such that $\sup _{x \in \mathbb{K}} p(f(x)-g(x))<\frac{\varepsilon}{2}$. But, again by definition, $F^{\mathrm{E}}(u)=E E F(U)$ (with the topology of uniform convergence on compact subsets of $(u)$. Without loss of generality, we may assume $u \in U$, and hence the a.p. of $F(u)$ or of $E$ and one direction of Schwartz's theorem from the introduction imply that $E \otimes F(U)$ is dense 
BIERSTEDT

in $F^{E}(u)$. Therefore we can find $h \in E \otimes F(u)$ with $\sup _{x \in K} p(g(x)-h(x))<\frac{\varepsilon}{2}$. Now $\left.h\right|_{K} \in E \otimes H_{F}(K)$ holds and $\sup _{x \in K} p(f(x)-h(x))<\varepsilon$, which proves the required density of $E \otimes H_{F}(K)$ in $H_{F}(K, E)$.

(2) is then clear from Schwartz's criterion because the space on the right hand side of the equation is nothing but $E \in H_{F}(K)$ - as a close look will immediately reveal.

In other words, it is always true (under the assumption of 4) that a function $\mathrm{f} \in \mathrm{C}(\mathrm{K}, \mathrm{E})$ which can be approximated uniformly on $\mathrm{K}$ by functions extending to elements of $F^{E}$ on open neighbourhoods of $K$ may also be approximated uniformly on $K$ by functions of the form

$$
h(x)=\sum_{i=1}^{n} e_{i} h_{i}(x) \text { for ail } x \in K
$$

$n \in \mathbb{N}$ finite (depending on $h$ ), $e_{i} \in E$, and $g_{i} \in H_{F}(k), i=1, \ldots, n$. But the a.p. of $H_{F}(K)$ is equivalent to the fact that, for arbitrary Banach space $E$, each function $f \in C(K, E)$ with the property that, given any $e^{\prime} \in E^{\prime}, e^{\prime}$ of may be approximated uniformly on $k$ by (scalar) functions belonging to $F$ on open sets containing $K$ is already an element of $H_{F}(K, E)$, i.e. can be approximated uniformly on $K$ by $E$-valued functions belonging to $F^{E}$ on open sets containing $K$. Or, to put it this way, $H_{F}(K)$ has the a.p. if and only if, given any Banach space $E$ and an arbitrary function $f \in C(K, E)$ as above, there exists for any $\varepsilon>0$, uniformly for all $e^{\prime}$ in the unit ball $E_{1}^{\prime}$ of $E^{\prime}$, an open set $u_{0} \supset \mathrm{K}$ and a function $g_{0}: u_{0} \rightarrow E$ continuous with $e^{\prime} \circ g_{0} \in F\left(u_{0}\right)$ for each $e^{\prime} \in E_{i}^{\prime}$ such that

$$
\left|\left(e^{\prime} \circ f\right)(x)-\left(e^{\prime} \circ g_{0}\right)(x)\right|<\varepsilon \text { for all } x \in K \text { and all } e^{\prime} \in E_{1}^{\prime} \text {. }
$$

REMARK: The description of $E \in H_{F}(K)$ as the right side of the equation in 4.(2) is of course independent of the hypothesis on $F$ in 4 , 
and so is the inclusion $H_{F}(K, E) \subset E \in H_{F}(K)$ which follows from this description. Hence, as obviously $E \theta_{\varepsilon} \mathrm{H}_{F}(\mathrm{~K})$ is always a topological linear subspace of $\mathrm{H}_{F}(K, E)$, we have

$$
\mathrm{E} \check{\otimes}_{\varepsilon} \mathrm{H}_{F}(\mathrm{~K}) \subset \mathrm{H}_{F}(\mathrm{~K}, E) \subset \mathrm{E} \varepsilon \mathrm{H}_{F}(\mathrm{~K})
$$

whenever $E$ is complete. So, by Schwartz's theorem, the a.p. of $\mathrm{H}_{F}(\mathrm{~K})$ clearly implies the equality

$$
\mathrm{H}_{F}(\mathrm{~K}, \mathrm{E})=\mathrm{E} \stackrel{\vee}{\otimes} \stackrel{\mathrm{V}}{\mathrm{H}}_{F}(\mathrm{~K})
$$

for complete $\ell . c$. spaces $E$, even without the hypothesis of 4.

Let $\mathrm{E}$ be complete and let the assumption of $4 .(1)$ be satisfied [or let $H_{F}(K)$ have the a.p.]. Then the preceding two theorems imply:

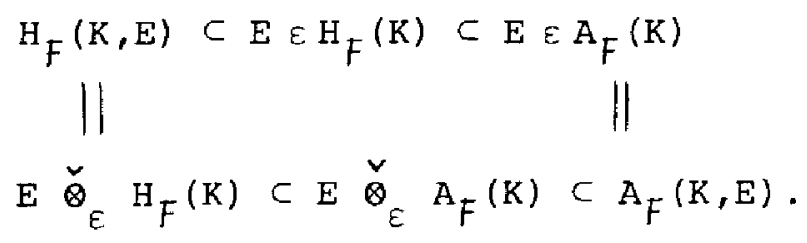

So we obtain from Schwartz's theorem:

5. COROLLARY: Let us again assume that, for some basis $\pi$ of neighbourhoods of $\mathrm{K}, \mathrm{F}(U)$ has the a.p. for each $u \in i r$, and let $A_{F}(K)=H_{F}(K)$ be valid.

Then $A_{F}(K)=H_{F}(K)$ has the a.p. if and only if $A_{F}(K, E)=H_{F}(K, E)$ holds for all complete l.c. Ior, equivalently, for all Banachl spaces $E$.

If, in concrete examples, one examines the methods that lead to a proof of $A_{F}(K)=H_{F}(K)$, it turns out very aften that these methods also prove $A_{F}(K, E)=H_{F}(K, E)$ for, at least, arbitrary Banach spaces E. Corollary 5 shows that it suffices to prove the equality ${ }_{F}(K, E)=H_{F}(K, E)$ for all Banach spaces $E$ to obtain both the a.p. of 
$A_{F}(K)=H_{F}(K)$ and $A_{F}(K, E)=H_{F}(K, E)$ even for arbitrary complete $l . c$. spaces $E$. On the other hand, sometimes the methods used in proving ${ }_{F}(K)={ }_{F}(K)$ may also be adapted to yield a direct proof of the a.p. of this space, and then $A_{F}(K, E)=H_{F}(K, E)$ holds for all complete lc. spaces by Corollary 5, too. In fact, Corollary 5 demonstrates that the two approaches which we have just outlined are equivalent.

REMARK: Similarly, if $E$ is a complete $\ell . c$. space and if $A_{F}(K)=H_{F}(K)$, then the a.p. of $E$ or of $A_{F}(K)=H_{F}(K)$ also implies $A_{F}(K, E)=H_{F}(K, E)$

\section{APPROXIMATION ON PRODUCT SETS}

Let us now turn to a description of the $\varepsilon$-product resp. complete $\varepsilon$-tensor product of two (or more) spaces of type $A_{F}(K)$ resp. $H_{F}(K)$. Such a description follows easily from the (well-known) general"slice product theorem" for subspaces of, say, $C\left(K_{I} \times K_{2}\right)$. (This siice product theorem was first stated in Eifler [17], but he points out that the result is already implicitly contained in Grothendieck [21]. For more general slice product theorems, for some ideas connected with the underlying method, and for more applications compare [4] and [5].) So let $x_{1}$ and $x_{2}$ be two completely regular (Hausdorff) spaces such that $x_{1} \times x_{2}$ is a $k_{I R}$-space. Then both $x_{1}$ and $x_{2}$ must be $k_{\mathbb{R}}$-spaces, and, on the other hand, $x_{1} \times x_{2}$ is $k_{\mathbb{R}}$, if both $x_{1}$ and $x_{2}$ are and if at least one of the spaces $x_{1}, x_{2}$ is even locally com pact (or if both $x_{1}$ and $x_{2}$ are hemicompact $k_{\mathbb{R}}$-spaces). Let $F_{1}$ resp. $F_{2}$ denote closed l.c. subsheaves of $c_{X_{1}}$ resp. $c_{X_{2}}$. Then we sets of completely regular $k$. proposition on k-spaces in IR-spaces, cf.[12], instead of Arhangel'skil's sheaf $F_{1} \in F_{2}$ " on $x_{1} \times x_{2}$ exists: 
$F_{1} \in F_{2}$ is uniquely determined by the following recuirements:

For all open subsets $u_{i} \subset x_{i}(i=1,2),\left(F_{1} \varepsilon F_{2}\right)\left(u_{1} \times u_{2}\right)=F_{1}\left(u_{1}\right) \varepsilon F_{2}\left(u_{2}\right)$, and, for all open $u_{i} \supset v_{i}$ on $x_{i}(i=1,2)$,

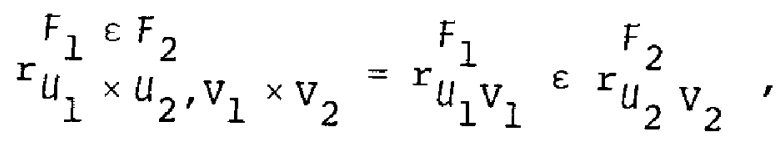

where $r_{U V}^{F}$ denotes the canonical restriction mapping $F(U) \rightarrow F(V)$ with respect to the sheaf $F$ and where the $\varepsilon$-product of continuous linear mappings is defined in, say, [7].

Let us now introduce the following notation: $\pi_{i}$ is the canonical projection of $x_{1} \times x_{2}$ onto $x_{i}(i=1,2)$, and, for arbitrary open subsets $u$ of $x_{1} \times x_{2}$,

$$
u_{t}:=\left\{x \in x_{2} ;(t, x) \in u\right\}\left(t \in x_{1}\right), u^{x}:=\left\{t \in x_{1} ;(t, x) \in u\right\}\left(x \in x_{2}\right) .
$$

Then we get a general description of $F_{1} \varepsilon F_{2}$ on open sets $u \subset x_{1} \times x_{2}$ as follows:

$$
\begin{aligned}
&\left(F_{1} \in F_{2}\right)(u):=\{f \in C(u) ; f(t, \cdot) \in F_{2}\left(u_{t}\right) \text { for each } t \in \pi_{1}(u) \text { and } \\
&\left.f(\cdot, x) \in F_{1}\left(u^{x}\right) \text { for each } x \in \pi_{2}(u)\right),
\end{aligned}
$$

endowed with the topology co of uniform convergence on compact subsets of $U$, and the canonical restriction mappings of the sheaf $F_{1} \varepsilon F_{2}$ are just the ordinary restrictions of functions. $F_{1} \varepsilon F_{2}$ is a closed

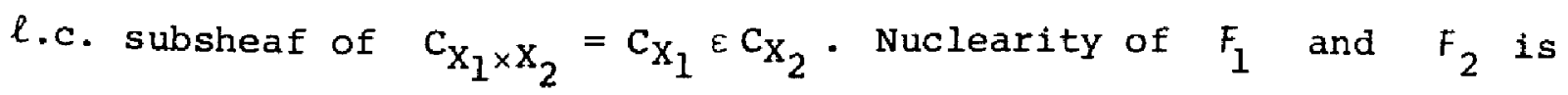
inherited by $F_{1} \in F_{2}([10], 1.2 \mathrm{c})$.

6. THEOREM: Let $\mathrm{k}_{\mathbf{i}}$ be a compact subset of $\mathrm{x}_{\mathbf{i}}(\mathbf{i}=1,2)$. Then we have: 
(1)

$$
\begin{aligned}
& A_{F_{1}}\left(K_{1}\right) \in A_{F_{2}}\left(K_{2}\right)=\left\{f \in C\left(K_{1} \times K_{2}\right) ; f(t, \cdot) \in A_{F_{2}}\left(K_{2}\right)\right. \text { and } \\
& \left.f(\cdot, x) \in A_{F_{1}}\left(K_{1}\right) \text { for all }(t, x) \in K_{1} \times K_{2}\right\} \\
& =\left\{f \in C\left(K_{1} \times K_{2}\right) ;\left.f(t, \cdot)\right|_{K_{2}} ^{0} \in F_{2}\left(\stackrel{0}{K}_{2}\right) \text { and }\left.f(\cdot, x)\right|_{K_{1}} ^{0} \in F_{1}\left(K_{1}\right)\right. \\
& \text { for all } \left.(t, x) \in K_{1} \times K_{2}\right\},
\end{aligned}
$$

with the sup-norm of $\mathrm{C}\left(\mathrm{K}_{1} \times \mathrm{K}_{2}\right)$,

(2)

$$
\begin{aligned}
& { }_{F_{1}}\left(K_{1}\right) \in H_{F_{2}}\left(K_{2}\right)=\left\{f \in C\left(K_{1} \times K_{2}\right) ; f(t, \cdot) \in \mathrm{H}_{F_{2}}\left(K_{2}\right)\right. \text { and } \\
& \left.f(\cdot, x) \in \mathrm{H}_{F_{I}}\left(K_{1}\right) \text { for all }(t, x) \in \mathrm{K}_{1} \times \mathrm{K}_{2}\right\} \\
& =\left\{\mathrm{f} \in \mathrm{C}\left(\mathrm{K}_{1} \times \mathrm{K}_{2}\right) ; \mathrm{f}(\mathrm{t}, \cdot)\right. \text { may be approximated uniformly on }
\end{aligned}
$$
$\mathrm{K}_{2}$ by functions belonging to $\mathrm{F}_{2}$ on open sets containing $\mathrm{K}_{2}$, and $\mathrm{f}(\cdot, \mathrm{x})$ may be approximated uniformly on $\mathrm{K}_{1}$ by functions belonging to $F_{1}$ on open sets containing $\mathrm{k}_{1}$ for each $\left.(t, x) \in \mathrm{K}_{1} \times \mathrm{k}_{2}\right\}$,

again with the sup-norm of $\mathrm{C}\left(\mathrm{K}_{1} \times \mathrm{K}_{2}\right)$, and:

(3) $\mathrm{H}_{F_{1}}\left(\mathrm{~K}_{1}\right) \check{\otimes}_{\varepsilon} \mathrm{H}_{F_{2}}\left(\mathrm{~K}_{2}\right) \subset \mathrm{H}_{F_{1}} \in F_{2}\left(\mathrm{~K}_{1} \times \mathrm{K}_{2}\right) \subset \mathrm{H}_{F_{1}}\left(\mathrm{~K}_{1}\right) \in \mathrm{H}_{F_{2}}\left(\mathrm{~K}_{2}\right)$

$$
\subset A_{F_{1}}\left(K_{1}\right) \in A_{F_{2}}\left(K_{2}\right) \subset A_{F_{1}} \varepsilon F_{2}\left(K_{1} \times K_{2}\right) \text {. }
$$

PROOF: Parts (1) and (2) follow immediately from the slice product theorem for subspaces of $\mathrm{C}\left(\mathrm{K}_{1} \times \mathrm{K}_{2}\right)$ quoted above. To prove

$$
\mathrm{H}_{F_{1}}\left(\mathrm{~K}_{1}\right) \check{\varrho}_{\varepsilon} \mathrm{H}_{F_{2}}\left(\mathrm{~K}_{2}\right) \subset \mathrm{H}_{F_{1} \in F_{2}}\left(\mathrm{~K}_{1} \times \mathrm{K}_{2}\right) \text {, }
$$

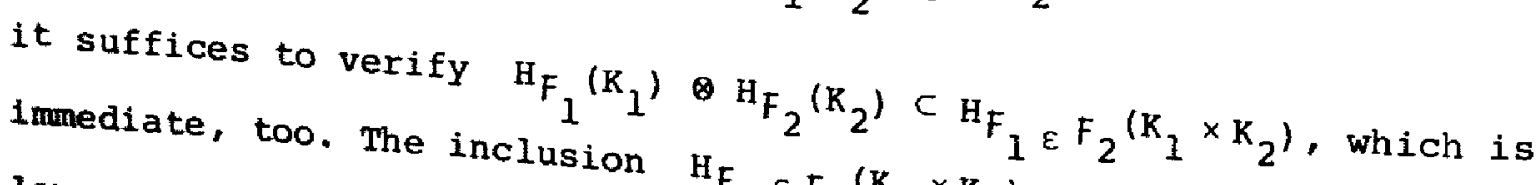
imediate, too. The inclusion $\mathrm{H}_{F_{1}} \varepsilon F_{2}\left(\mathrm{~K}_{1} \times \mathrm{K}_{2}\right) \subset \mathrm{H}_{F_{1}}\left(\mathrm{~K}_{1}\right) \varepsilon \mathrm{H}_{F_{2}}(\mathrm{~K})$ folfrom the description of the $\varepsilon$-production of the sheaf $F_{1} \varepsilon F_{2}$ and 
Finally $A_{F_{1}}\left(K_{1}\right) \in A_{F_{2}}\left(K_{2}\right) \subset A_{F} \varepsilon F_{2}\left(K_{1} \times K_{2}\right)$, because $\frac{a}{K_{1} \times K_{2}}=\stackrel{o}{K_{1}} \times \stackrel{o}{K_{2}}$ and hence (by the description of $F_{1} \in F_{2}$ ):

$$
{ }^{A} F_{1} \varepsilon F_{2}\left(K_{1} \times K_{2}\right)=\left\{f \in C\left(K_{1} \times K_{2}\right) ;\left.f\right|_{K_{1} \times K_{2}^{o}} \in\left(F_{1} \varepsilon F_{2}\right)\left(\stackrel{K}{1}_{1} \times \stackrel{o}{K_{2}}\right)=F_{1}\left(K_{1}\right) \varepsilon F_{2}\left(K_{2}\right)\right\}
$$

$=\left\{\mathrm{f} \in \mathrm{C}\left(\mathrm{K}_{1} \times \mathrm{K}_{2}\right) ;\left.\mathrm{f}(\mathrm{t}, \cdot)\right|_{\mathrm{K}_{2}} ^{0} \in \mathrm{F}_{2}\left(\stackrel{0}{\mathrm{~K}_{2}}\right)\right.$ and $\left.\mathrm{f}(\cdot, \mathrm{x})\right|_{\mathrm{K}_{1}} ^{0} \in \mathrm{F}_{1}\left(\stackrel{0}{\mathrm{~K}_{1}}\right)$ (only) for all

$$
\left.(t, x) \in \stackrel{0}{\mathrm{~K}_{1}} \times \stackrel{0}{\mathrm{~K}_{2}}\right\}
$$

As 6. (I) (together with the description of $A_{F_{1} \in F_{2}}\left(K_{1} \times K_{2}\right)$ at the end of the preceding proof) shows, $A_{F_{1}}\left(K_{1}\right) \varepsilon A_{F_{2}}\left(K_{2}\right)$ will in general be strictly contained in ${ }^{A} F_{1} \in F_{2}\left(K_{1} \times K_{2}\right)$, and it is easy to construct examples for this phenomenon. However, a simple topological assumption forces equality here, as part (2) of our next result demonstrates.

7. THEOREM: (1) Let, for some basis $u_{1}$ of neighbourhoods of $K_{1}, F_{1}\left(u_{1}\right)$ have the a.p. for each $u_{1} \in u_{1}$ or let, for some basis $u_{2}$ of neighbourhoods of $\mathrm{K}_{2}, \mathrm{~F}_{2}\left(u_{2}\right)$ have the a.p. for each $u_{2} \in U_{2}$. [Instead of this, we could also require $\mathrm{H}_{F_{1}}\left(\mathrm{~K}_{1}\right)$ or $\mathrm{H}_{\mathrm{F}_{2}}\left(\mathrm{~K}_{2}\right)$ to have the a.p.]. Then

$$
{ }^{H_{F}} \in F_{2}\left(K_{1} \times K_{2}\right)=H_{F_{1}}\left(K_{1}\right) \check{\otimes}_{\varepsilon} H_{F_{2}}\left(K_{2}\right) \text { holds. }
$$

(2) If $\mathrm{K}_{1}$ and $\mathrm{K}_{2}$ are "fat", i.e. satisfy $\overrightarrow{\mathrm{o}}_{i}=\mathrm{K}_{i} \quad(i=1,2)$, we get:

$$
A_{F_{1}}\left(K_{1}\right) \in A_{F_{2}}\left(K_{2}\right)=A_{F_{1}} \in F_{2}\left(K_{1} \times K_{2}\right)
$$

PROOF: (1) The remark in brackets is obvious from 6. (3) and Schwartz's theorem. For the proof of (1) under the assumption on $F_{1}$ resp. $F_{2}$ ' 
it suffices to show density of $\mathrm{H}_{F_{1}}\left(\mathrm{~K}_{1}\right) \otimes \mathrm{H}_{F_{2}}\left(\mathrm{~K}_{2}\right)$ in $\mathrm{H}_{F_{1} \in F_{2}}\left(\mathrm{~K}_{1} \times \mathrm{K}_{2}\right)$. So let $\mathrm{f} \in \mathrm{H}_{F_{1}} \in \mathrm{F}_{2}\left(\mathrm{~K}_{1} \times \mathrm{K}_{2}\right)$ and $\varepsilon>0$ be given and $\mathrm{find}$ an open set $U$ containing $K_{1} \times K_{2}$ and a function $g \in\left(F_{1} \varepsilon F_{2}\right)(u)$ such that

$$
\sup _{x \in K_{1} \times K_{2}}|f(x)-g(x)|<\frac{\varepsilon}{2} .
$$

Without loss of generality we may assume $u=u_{1} \times u_{2}$ with $u_{i} \in u_{i}$ $(i=1,2)$, and hence

$$
\left(F_{1} \varepsilon F_{2}\right)(u)=F_{1}\left(u_{1}\right) \in F_{2}\left(u_{2}\right)=F_{1}\left(u_{1}\right) \check{\otimes}_{\varepsilon} F_{2}\left(u_{2}\right)
$$

by Schwartz's theorem, because $F_{1}\left(u_{1}\right)$ or $F_{2}\left(u_{2}\right)$ has the a.p. Then there exists $h \in F_{1}\left(U_{1}\right) \otimes F_{2}\left(U_{2}\right)$ such that

$$
\sup _{x \in \mathrm{K}_{1} \times \mathrm{K}_{2}}|\mathrm{~g}(\mathrm{x})-\mathrm{h}(\mathrm{x})|<\frac{\varepsilon}{2} .
$$

Now $\left.\quad \mathrm{h}\right|_{\mathrm{K}_{1}} \times \mathrm{K}_{2} \in \mathrm{H}_{\mathrm{F}_{1}}\left(\mathrm{~K}_{1}\right) \otimes \mathrm{H}_{\mathrm{F}_{2}}\left(\mathrm{~K}_{2}\right)$ and $\sup _{x \in \mathrm{K}_{1} \times K_{2}}|f(x)-h(x)|<\varepsilon$.

(2) Notice that, by the identity $\mathrm{C}\left(\mathrm{K}_{1} \times \mathrm{K}_{2}\right)=\mathrm{C}\left(\mathrm{K}_{1}, \mathrm{C}\left(\mathrm{K}_{2}\right)\right)=\mathrm{C}\left(\mathrm{K}_{2}, \mathrm{C}\left(\mathrm{K}_{1}\right)\right)$, for arbitrary $f \in A_{F_{1}} \in F_{2}\left(K_{1} \times K_{2}\right), I_{1}: t \rightarrow f(t, \cdot) \operatorname{resp} \cdot I_{2}: x+f(\cdot, x)$ yield continuous linear mappings of $K_{1}$ resp. $K_{2}$ into $C\left(K_{2}\right)$ resp. $C\left(K_{1}\right)$. The characterization of $A_{F_{1}} \in F_{2}\left(K_{1} \times K_{2}\right)$ at the end of the proof of 6 implies $\left.I_{1}(\stackrel{\circ}{K})_{1}\right) \subset A_{F_{2}}\left(K_{2}\right)$ and $I_{2}\left(\stackrel{0}{K_{2}}\right) \subset A_{F_{1}}\left(K_{1}\right)$ and hence also $I_{1}\left(\overline{K_{1}}\right) \subset A_{F_{2}}\left(K_{2}\right)$ and $I_{2}\left(\overline{K_{2}}\right) \subset A_{F_{1}}\left(K_{1}\right)$. So, for fat sets $K_{1}$ and $K_{2}$, the assertion follows immediately from $6 .(1)$.

8. COROLLARY

these spaces ${ }^{A} F_{i}\left(K_{i}\right)={ }^{H} F_{i}\left(K_{i}\right)(i=1,2)$ holds and if one of these spaces has the a.p., then we obtain

$$
{ }_{F_{1}}^{\mathrm{H}_{1}} E F_{2}\left(\mathrm{~K}_{1} \times \mathrm{K}_{2}\right)=\mathrm{A}_{F_{1}}\left(\mathrm{~K}_{1}\right) \in \mathrm{A}_{F_{2}}\left(\mathrm{~K}_{2}\right) \text {. }
$$


So we get even ${ }_{F_{1}} \varepsilon F_{2}\left(\mathrm{~K}_{1} \times \mathrm{K}_{2}\right)=\mathrm{A}_{F_{1} \varepsilon F_{2}}\left(\mathrm{~K}_{1} \times \mathrm{K}_{2}\right)$ for fat sets $\mathrm{K}_{1}$ and $\mathrm{K}_{2}$.

(2) $\mathrm{H}_{F_{I}} \& F_{2}\left(\mathrm{~K}_{1} \times \mathrm{K}_{2}\right)$ has the a.p. whenever both $\mathrm{H}_{F_{1}}\left(\mathrm{~K}_{1}\right)$ and $\mathrm{H}_{F_{2}}\left(\mathrm{~K}_{2}\right)$ have the a.p.

(3) If $K_{1}$ and $K_{2}$ are fat and if both $A_{F_{1}}\left(K_{1}\right)$ and $A_{F_{2}}\left(K_{2}\right)$ have the a.p., ${ }^{A} F_{1} \in F_{2}\left(K_{1} \times K_{2}\right)$ has the a.p., too.

PROOF: (1) is clear from Schwartz's theorem, 6. (3), and 7. (2).

and (3) follow from 7 by aid of the result (Schwartz [26], Proposition 11, Corollaire 2) that the $\varepsilon$-product of two compete $\ell . c$. spaces with a.p. also enjoys the a.p.

Induction on 7 and $8 .(1)$ using, among other (obvious) things, that finite $\varepsilon$-products are associative and that $\varepsilon$-products of complete spaces with a.p. are again spaces with a.p. yields now:

9. COROLLARY: Let $\mathrm{x}_{1}, \ldots, \mathrm{x}_{\mathrm{n}}$ be completely regular (Haustorf6) spaces with $x_{1} \times \ldots \times x_{n}$ a $k_{\mathbb{R}}$-space, let $F_{1}, \ldots, F_{n}$ be closed l.c. subsheaves of $\mathrm{C}_{\mathrm{x}_{1}}, \ldots, \mathrm{C}_{\mathrm{X}_{\mathrm{n}}}$, and $\mathrm{K}_{1}, \ldots, \mathrm{K}_{\mathrm{n}}$ compact subsets of $\mathrm{x}_{1}, \ldots, \mathrm{x}_{\mathrm{n}}$, respectively.

(1) Let, for same basis $u_{i}$ of neighbourhoods of $k_{i}, F_{i}\left(u_{i}\right)$ have the a.p. for each $u_{i} \in U_{i}(i=1, \ldots, n$ except for at most one i) or let all but one $\mathrm{H}_{F_{i}}\left(\mathrm{~K}_{i}\right)(\mathrm{i}=1, \ldots, \mathrm{n})$ have the a.p. Then

${ }_{F_{1}} \varepsilon \ldots \varepsilon F_{n}\left(K_{1} \times \ldots \times K_{n}\right)=H_{F_{1}}\left(K_{1}\right) \check{\otimes}_{\varepsilon} \ldots \check{\otimes}_{\varepsilon} H_{F_{n}}\left(K_{n}\right)$

is true, and if all ${ }_{F_{F}}\left(\mathrm{~K}_{i}\right)(i=1, \ldots, n)$ have the a.p., the same holds for $\mathrm{H}_{\mathrm{F}_{1}} \in \ldots \in \mathrm{F}_{\mathrm{n}}\left(\mathrm{K}_{1} \times \ldots \times \mathrm{K}_{\mathrm{n}}\right)$.

(2) Let all the sets $\mathrm{K}_{1}, \ldots, \mathrm{K}_{\mathrm{n}}$ be fat. Then

$$
A_{F_{1}} \varepsilon \ldots \varepsilon F_{n}\left(K_{1} \times \ldots \times K_{n}\right)=A_{F_{1}}\left(K_{1}\right) \varepsilon \ldots \varepsilon A_{F_{n}}\left(K_{n}\right)
$$


holds true, and if all ${ }^{A_{F_{i}}}\left(K_{i}\right)(i=1, \ldots, n)$ have the a.p., the same holds for $A_{F_{1}} \in \ldots \varepsilon F_{n}\left(K_{1} \times \ldots \times K_{n}\right)$.

(3) Let, for each $i=1, \ldots, n, K_{i}$ be fat and $A_{F_{i}}\left(K_{i}\right)=H_{F_{i}}\left(K_{i}\right)$. If then all these spaces (except for at most onel have the a.p., ${ }^{A} F_{I} \varepsilon \ldots \varepsilon F_{n}\left(K_{1} \times \ldots \times K_{n}\right)=H_{F_{l}} \varepsilon \ldots \varepsilon F_{n}\left(K_{l} \times \ldots \times K_{n}\right)$ is valid, too.

For the corresponding spaces of functions with values in a quasicomplete $\ell . c$. space $E$ (see section 1), we get e.g.:

10. COROLLARY: (1) Let $\mathrm{K}_{1}, \ldots, \mathrm{K}_{\mathrm{n}}$ be fat. Then

$$
A_{F_{1}} \varepsilon \ldots \varepsilon F_{n}\left(K_{1} \times \ldots \times K_{n}, E\right)=E \varepsilon A_{F_{l}}\left(K_{1}\right) \varepsilon \ldots \varepsilon A_{F_{n}}\left(K_{n}\right)
$$

is true.

(2) Let $\mathrm{E}$ be complete and let, for some basis $\pi_{i}$ of neighis valid.

(3) Let $E$ be complete, let $\mathrm{K}_{i}$ be fat and $A_{F_{i}}\left(K_{i}\right)=H_{F_{i}}\left(K_{i}\right)$ for each $i=1, \ldots, n$. Then if ale the spaces $A_{F_{i}}\left(K_{i}\right)=H_{F_{i}}\left(K_{i}\right)$ ${ }^{A} F_{1} \varepsilon \ldots \varepsilon F_{n}\left(K_{1} \times \ldots \times K_{n}, E\right)=H_{F_{1}} \varepsilon \ldots \varepsilon F_{n}\left(K_{1} \times \ldots \times K_{n}, E\right)$ holds, too.

PROOF :

(1) is a consequence of under the hypothesis of (2) $3 .(1)$ and $9 .(2)$. Let us remark that, ( $\left.F_{1} \varepsilon \ldots \varepsilon F_{n}\right)(U)$ (as $\varepsilon$-product of complete 
spaces with a.p.) satisfies the a.p. for each $u$ in the basis

$$
u^{\mathrm{p}}:=\left\{u_{1} \times \ldots \times u_{n} ; u_{i} \in U_{i} \quad(i=1, \ldots, n)\right\}
$$

of neighbourhoods of $k_{1} \times \ldots \times k_{n}$. Hence (2) follows from $4 .(1)$ and 9. (1). Finally (3) is implied by 9. (2), (3) and by the remark at the very end of section 1 . $\square$

\section{DISCUSSION OF THE MOTIVATING EXAMPLES}

In this final section, we will look at some of the known results in the case of our motivating examples of sheaves $F$ (cf. 1 above) and will point out that, between some theorems in the literature, strong relations follow from our previous discussion. It is not intended here to survey all the relevant articles, but we will rather illustrate some of the ideas which might play a rôle, when one tries to apply the results of sections 1 and 2 , by specific examples.

Perhaps the case most people have been interested in is $F=0$, the nuclear Frëchet sheaf of holomorphic functions on a complex manifold $x$. For simplicity, however, we will only deal with holomorphic functions on $x=\mathbb{C}^{N}(N \geq 1)$ here. It is clear that finite $\varepsilon$-products of sheaves 0 are nothing but the correspending sheaf 0 on the product and that, for any quasi-complete $\ell . c$. space $E, O^{E}$ is just the sheaf of E-valued holomorphic functions. When $F=0$, we will write, for short, $A(K, E), H(K, E)$ instead of $A_{F}(K, E), H_{F}(K, E)$, respectively.

For $F=0$, some of the results in sections 1 and 2 are apparently part of the "folklore" of the subject, but usually not easily accessible in the literature: We have already pointed out in the introduction that this paper is based on a generalization of the "old" article [2]. Later on (in [1], section 1), O. B. Bekken looked at closed subspaces of $C(K), K$ compact, with the so-called "slice property" 
and showed that this property implies the a.p. After the proper change of notation and some identifications (using the fact that each Banach space is a closed subspace of $C^{\left(K^{\prime}\right)}$ for some compact $\left.K^{\prime}\right)$ his results there are quite similar to our theorem 3 (for Banach spaces $E$ ). In section 3 of [1], (making use of the nuclearity of 0 ) Bekken obtains a proposition related to (but somewhat weaker than) our theorem 4. For a detailed account of the relation of the slice property with the a.p. and the consequences of a theorem of llilne in this connection, see also $(6)$.

As usual with spaces of holomorphic functions, we must now split up our discussion for the cases $N=1$ and $N \geq 2$. If $N=1$ i.e. $K$ is a compact subset of the complex plane, the problem is completely solved: $A(K)$ and $H(K)$ have then always the a.p. (whereas it remains an open problem whether even the Banach algebra $H^{\infty}(D)$ of all bounded holomorphic functions on the open unit disk $D$ enjoys the a.p. Remark that the a.p. of the disk algebra $A(\bar{D})=H(\bar{D})$ is really quite trivial :). This interesting result is due to the joint effort of several people (and also, unfortunately, not easily accessible in the literature in its full generality): Eifler [17], Gamelin-Garnett [19], section 6 for $H(K)$, and Davie [15] for $A(K)$ (they all use vector -valued results). More generally, Gamelin [18], section 12 has pointed out that the constructive techniques (and the approximation scheme) of Mergelyan and vitushkin show that the so-called "T-invariant"algebras have the a.p. As to $A(K)=H(K)$ in the case of one variable, a necessary and sufficient condition (involving continuous analytic capacity) was given by Vitushkin, see e.g. [19] and [29].

For $N \geq 2$, there are only partial results. Remark first that, by an example of Diederich and Fornaess, there exists a relatively compact domain $G$ of holomorphy in $\mathbb{C}^{N}$ with $C^{\infty}$-boundary such that $A(K) \neq H(K)$ for $K=\bar{G}$. For a survey of some related recent work on 
the question when $A(K)=H(K)$ in several complex variables, we refer to Birtel [11], and for results in "finite S. Ps. C. manifolds" to Rossi-Taylor [25].

It is known now that $A(K)$ (or $H(K)$ ) has the a.p. for the following types of compact sets $K \subset \mathbb{C}^{\mathrm{N}}$ :

(i) $\mathrm{K}$ is the closure of a strictly pseudoconvex region with sufficiently smooth (say, $\mathrm{C}^{3}$-) boundary, or:

(ii) $\mathrm{K}$ is the closure of a regular weil polyeder.

Both conditions imply $K$ fact (trivially), and $A(K)=H(K)$ (in case ( $i)$, this approximation theorem is due to Henkin-Lieb-Kerzman, in case (ii), it is a result of Petrosjan). (i) was proved e.g. in Bekken [1], section 2, applying a vector-valued version of Henkin's separation of singularities result. It also follows from sibony [27], Proposition 4 (in view of our Corollary 5). Sibony [27], p. 173 has also remarked that Petrosjan's arguments may be modified to yield $A(K, E)=H(K, E)$ for each Fréchet space $E$ if $K$ is the closure of a regular weil polyeder, and hence (ii) follows again from ourcorollary 5.

REMARK: The method of "localization of the a.p." for certain function spaces (cf. [5] and [10]) may be used to show that $A\left(K^{\prime}\right)$ has the a.p. for compact sets $K^{\prime}$ that are "sufficiently well" disjoint unions of sets $K$ as above and that some related function spaces have the a.p., too (cf. [5], Corollary 15), but we will not go into details here.

Let us now explicitly state what we get from the preceding results by applying our Corollaries 9 and 10 :

14. THEOREM: (I) $\mathrm{H}(\mathrm{K})$ has the a.p. if $\mathrm{K}=\mathrm{K}_{1} \times \ldots \times \mathrm{K}_{\mathrm{n}}$. with $\mathrm{k}_{\mathrm{i}}$ $(i=1, \ldots, n)$ either

(i) any compact subset of $\boldsymbol{c}$ or 
(ii) the closure of a strictly pseudoconvex region with sufficiently smooth boundary or of a regular weil polyeder.

(2) $A(K)$ has the a.p. under the same conditions if one requires in $(1)(i)$, additionally, $K_{i}$ to be fat. And $A(K)=$ $=A\left(K_{1}\right) \check{\otimes}_{\varepsilon} \ldots \check{\otimes}_{\varepsilon} A\left(K_{n}\right)$ is then true.

(3) $\mathrm{H}(\mathrm{K})=\mathrm{A}(\mathrm{K})$ holds for $\mathrm{K}=\mathrm{K}_{1} \times \ldots \times \mathrm{K}_{\mathrm{n}}$ with $\mathrm{K}_{\mathrm{i}}(\mathrm{i}=1, \ldots, \mathrm{n})$ either $(i)$ a bat compact set in $\mathbb{c}$ with $\mathrm{H}\left(\mathrm{K}_{\mathrm{i}}\right)=\mathrm{A}\left(\mathrm{K}_{\mathrm{i}}\right)$ or as in (1)(ii) above.

Let then $\mathrm{E}$ be an arbitrary complete l.c. space.

(4) Under the assumptions of (2),

$$
A(K, E)=E \check{\otimes}_{E} A\left(K_{l}\right) \check{\otimes}_{E} \ldots \check{\otimes}_{E} A\left(K_{n}\right) \text { holds. }
$$

(5) Under the assumptions of $(3)$, we have $\mathrm{A}(\mathrm{K}, \mathrm{E})=\mathrm{H}(\mathrm{K}, \mathrm{E})$, too.

11. (3) is related to a result of weinstock [30], p. 812, where, instead of the assumption of a smooth boundary in 11.(1)(ii), he needs only the so-called "segment property" of $\mathrm{K}$. (Weinstock's methods are quite different, however.) At this point, a few remarks on sibony's paper [27] are also in order (in connection with our preceding results):

Proposition 1 of [27] is, in some sense, easy, if not trivial, as our theorem $4 .(1)$ (and its simple proof) demonstrates: It is not necessary to invoke Gleason's theorem at this point; the well-known nuclearity (or even the a.p.) of 0 and simple tensor product argu ments suffice: Corollaire 3 of [27] corresponds with $7 .(1)$ and 10.(2) in this paper. As we have already noted above, however, sibony's proposition 4 is really a non-trivial result based on Henkin's method it is (essentially) equivalent to theorem 2.4 of Bekken [1]. Finally, Corollaire 8 of [27] corresponds with our theorem 11. (5). It should perhaps be pointed out that, whereas part of sibony's proofs looks as 
though they are based on theorems and methods which are just true in his given special situation, it turns out from our discussion above that what is really needed is only a proof of the a.p. of $A(K)(=H(K))$ to make everything work, even in many other cases.

We turn to sheaves $F$ of harmonic functions or, more generally, of null-solutions of hypoelliptic differential operators with $C^{\infty}$-coefficients now. These are again nuclear Fréchet sheaves, and hence our assumption that $F$ has the a.p. is certainly satisfied. For nuclearity of the sheaves in axiomatic potential theory, cf. ConstantinescuCornea $[14], \S 11$.

In this case, we will assume for the moment that the compact set $K$ is the closure of some open subset $U$ of $X$ (and hence fat). A very nice phenomenon may occur here which yields a completely trivial solution to the question of the a.p. for $A_{F}(K)$ : If we suppose that $U$ is a regular set for the Dirichlet problem with respect to the sheaf $F, i . e$. for each $g \in C(\partial U)$ there exists a unique function $f \in A_{F}(\bar{u})$ with $\left.f\right|_{\partial U}=g$, the continuous linear restriction mapping $L:\left.f \rightarrow f\right|_{\partial U}$ is bijective from $A_{F}(K)$ onto $C(\partial U)$ and hence yields a topological isomorphism of these Banach spaces. (A maximum principle for functions in $A_{F}(K)$ will imply that $L$ is even an isometry.) Then $A_{F}(K)$ certainly has the a.p. In fact, it would be enough for such a result that $L:\left.f \rightarrow f\right|_{K}$ is bijective from $A_{F}(K)$ onto a closed subspace with $a, p$. of $C\left(K^{\prime}\right)$ for some closed subset $K^{\prime}$ of $K$ (say, $a$ closed set $K^{\prime} \subset \partial(u)$.

Let for instance $F$ be the sheaf $\pi$ of (real) harmonic functions on $\mathbf{R}^{n}(n \geq 2)$. We refer e.g. to Ho-Van-Thi-Si [22], p. 617/8, $621 / 2,626,637$ for conditions concerning, say, the equalities

(i) $\quad A_{\mathscr{X}}(K)=H_{\mathscr{X}}(K)$, and

(ii) $\left.A_{\mathcal{X}}(K)\right|_{\partial K}=C(\partial K)$ (or, equivalently, by the maximum principle, $L: A_{\mathcal{X}}(K) \rightarrow C(\partial K)$ bijective and isometric). 
Let us only note that in general a suitable (outside) cone condition implies both (i) and (ii) and that, in the case $n=2$, (i) and (ii) are valid for a compact set $k$ such that each point $x \in \partial K$ is a boundary point of a connected component of the complement of $K$. So then $A_{\mathcal{K}}(K)=H_{\mathscr{K}}(K)$ has the a.p.

We also refer to Weinstock [31] for results on $A_{F}(K)=H_{F}(K)$ for sheaves $F=N_{L}$ (on $\mathbb{R}^{n}$ ) of null solutions of (linear) elliptic partical differential operators $L$ of order $m$ with constant coefficients in this connection and to Vincent-Smith [28] for $A_{F}(K)=H_{F}(K)$ in the setting of harmonic sheaves $F$ of axiomatic potential theory. It would lead us too far afield even to give only complete references for all interesting relevant results in this direction.

Another argument then yields the a.p. of ${ }^{A_{F}}(\mathrm{~K})$ and $\mathrm{H}_{F}(\mathrm{~K})$ even in a much more general setting:

12. THEOREM: Let again $\pi$ be the sheaf of harmonic functions on $\mathbb{R}^{n}$ $\left(\mathrm{n} \geq 2\right.$ ) and $\mathrm{K}$ an arbitrary compact subset of $\mathbf{R}^{\mathrm{n}}$.

(1) Then both $A_{\mathcal{H}}(K)$ and $\mathrm{H}_{\mathcal{H}}(\mathrm{K})$ always have the a.p.

(2) Hence $A_{\mathcal{T}}(\mathrm{K}, \mathrm{E})=E \check{\ddot{\theta}}_{E} A_{\mathcal{H}}(\mathrm{K})$ holds for each completel.c. space $E$, and, for such an $E, A_{\mathcal{H}}(K)=H_{\mathcal{H}}(K)$ always implies A-maximal measure (or, equid null measure is the only Choquet boundary of A) concentrated in the [16], p. 99) that the state is order isometric to $S=S(A)$ is a simplex and that $A$ tions in $C(S)$. However. $A(S)$ of all affine func$A(S)$ has the a.p.: In fact (L) - space. (This 
argument can be found e.g. in the proof of Corollary 2.6, p. 477 of Namioka-Phelps [23].) (2) follows from (1) and 3.(2), 5 above.

For the connection between simplicial spaces and the solution of "weak Dirichlet problems" see Effros-Kazdan [16]: $\mathrm{A}_{\mathscr{H}}(\mathrm{K})$ (say) is simplicial if and only if each continuous function defined on a compact subset of the choquet boundary of $A_{\mathcal{H}}(K)$ may be extended to an element of $A_{\mathcal{H}}(K)$ of the same norm.

But now we get the a.p. of $A_{F}(K)$ and $H_{F}(K)$ for many sheaves $F$ of axiomatic potential theory and all sets $\mathrm{K}=$ closure of a relativeiy compact open set $u$ : In fact, under certain axions on the underlying harmonic space $(X, F)$, it is known that $A_{F}(K)$ resp. $H_{F}(K)$ is again simplicial, and then we may proceed as in the proof of theorem 12 to carry the corresponding results over to this (much more general) setting. For the relevant axioms needed here and the $A_{F}(K)$ resp. $H_{F}(K)$ is a simplicial space, we refer to Effros-Kazu. [16], Cor. 4.3, p. 108 and Cor. 4.2, p. 112. (For a necessary and sufficient condition for $A_{F}(K)=H_{F}(K)$ in this setting see [16], theorem 4.4). In [16], the axioms still excluded general open sets $u$ for degenerate elliptic equations, but the corresponding problem was solved affirmatively by Bliedtner-Hansen [13], and we refer to [13] for the most general results on simplicial spaces $A_{F}(K)$.

In concluding, we should point out that the $\varepsilon$-product $\mathbb{H}_{1} \varepsilon \mathbb{K}_{2}$ of two sheaves of harmonic functions in axiomatic potential theory yields nothing but the (multiply resp.) separately harmonic functions of Gowrisankaran [20] resp. Reay [24]. We leave it to the reader to combine our preceding remark on the a.p. of ${ }_{F}(K)$ resp. $H_{F}(K)$ in axiomatic potential theory with the results in section 2 above to obtain, say, theorem 11 and lemma 23 of [24] without any effort. Of course, we could also immediately state results for "mixed" (say) holomorphic-harmonic sheaves $0 \varepsilon \pi$ etc., but the preceding examples 
and applications may suffice.

\section{REFERENCES}

[1] O. B. BEKKEN, The approximation property for Banach spaces of analytic functions, preprint (1974), unpublished.

[ 2] K.-D. BIERSTEDT, Function algebras and a theorem of Mergelyan for vector - valued functions, Papers from the summer Gathering on Function Algebras, Aarhus, Various Publication Series 9 (1969), 1-10.

[ 3] K.-D. BIERSTEDT, Gewichtete Räume stetiger vektorwertiger Funktionen und das injektive Tensorprodukt I, II, J. reine angew. Math. 259 (1973), 186-210; 260 (1973), 133-146.

[ 4 ] K.-D. BIERSTEDT, Injektive Tensorprodukte und Slice-produkte gewichteter Räume stetiger Funktionen, J, reine angew. Math, 266 (1974), 121-131.

[ 5] K.-D. BIERSTEDT, The approximation property for weighted function spaces; Tensor products of weighted spaces, Function Spaces and Dense Approximation (Proc. Conference Bonn 1974), Bonner. Math. Schriften 81 (1975), 3-25; 26-48.

[ 6 ] K.-D. BIERSTEDT, Neuere Ergebnisse zum Approximationsproblem von Banach-Grothendieck, Jahrbuch Überblicke Math. 1976,

[ 7] K.-D. BIERSTEDT and R. MEISE, Lokalkonvexe Unteräume in topologischen Vektorräumen und das $\varepsilon$-Produkt, Manuscripta tionseigenschaft lokalkonvexer Funktionenräume, Math.
Ann. 209 (1974), 99-107. 
[ 9] K.-D. BIERSTEDT, B. GRAMSCH and R. MEISE, Lokalkwexe Garben und gewichtete induktive Limites F-morpher Funktionen, Function Spaces and Dense Approximation (Proc. Conference Bonn 1974), Bonner Math. Schriften 81 (1975), 59- 72.

[10] K.-D. BIERSTEDT, B. GRAMSCH and R. MEISE, Approximationseigenschaft, Lifting und Ko-homologie bei lokalkonvexen Produktgarben, Manuscripta Math. 19 (1976), 319-364.

[1]] F. T. BIRTEL, Holomorphic approximation to boundary value algebras, Bull. Amer. Math. Soc. 84 (1978), 406-416.

[12] J. L. BLASCO, Two problems on $k_{\mathbb{R}}$-spaces, preprint (1977), to appear in Acta Math. Sci. Hungar.

113] J. BLIEDTNER and W. HANSEN, Simplicial cones in potential theory, Inventiones Math. 29 (1975), 83-110.

[14] C. CONSTANTINESCU and A. CORNEA, Potential theory on harmonic spaces, Springer Grundlehren der Math. Wiss. Band 158 (1972).

[15] A. M. DAVIE, The approximation property of $A(K)$ on plane sets, private communication (1969), unpublished.

[16] E. G. EFFROS and J. L. KAZDAN, Applications of Choquet simplexes to elliptic and parabolic boundary value problems, J. Diff. Equations 8 (1970), 95-134.

[17] L. EIFLER, The siice product of function algebras, Proc. Amer. Math. Soc. 23 (1969), 559-564.

[18] T. W. GAMELIN, Uniform approximation on plane sets, Approximation Theory (Editor: G. G. Lorentz), Academic Press, (1973), 101-149.

[19] T. W. GAMELIN and J. GARNETT, Constructive techniques in rational approximation, Trans. Amer. Math. Soc. 143 (1969), $187-200$. 
[20] K. GOWRISANKARAN, Multiply harmonic functions, Nagoya Math. J. $28(1966), 27-48$.

[2I] A. GROTHENDIECK, Produits tensoriels topologiques et espaces nucléaires, Memoirs Amer. Math. Soc. 16 (1955), reprint (1966).

[22] HO-VAN-THI-SI, Frontière de Choquer dans les espaces de fonctions et approximation des fonctions harmoniques, Bull. Soc. Roy Sci. Liège 41 (1972), 607-639.

[23] I. NAMIOKA and R. R. PHELPS, Tensor products of compact convex sets, Pacific J. Math. 31 (1969), $469-480$.

[24] I. REAY, Subduals and tensor products of spaces of harmonic functions, Ann. Inst. Fourier 24 (1974), $119-144$.

[25] H. ROSSI and J. L. TAYLOR, On algebras of holonorphic functions on finite pseudoconvex manifolds, J. Functional Anal. 24 (1977), 11-31.

[26] L. SCHWARTz, Thēorie des distributions à valeurs vectorielles I, Ann. Inst. Fourier 7 (1957), 1-142.

[27] N. SIBONY, Approximation de fonctions à valeurs dans un Fréchet par des fonctions holomorphes, Ann. Inst. Fourier 24 $(1974), 167-179$.

[28] G. F. VINCENT-SMITH, Uniform approximations of harmonic functions, Ann. Inst. Fourier 19 (1969), 339-353.

[29] A. G. VITUSHKIN, Uniform approximation by holomorphic functions, J. Functional Anal. 20 (1975), 149-157.

[30] B. M. WEINSTOCK, Approximation by holomorphic functions on certain product sets in $\mathbf{c}^{\mathrm{N}}$. Pacific J. Math. 43 (1972),
$811-822$. 